\title{
Enfermeras de salud mental: de dónde venimos y dónde estamos
}

\author{
Mental Health Nurses: Where We Come from and Where We Are Today
}

\section{Julián Carretero Román}

Enfermero de salud mental. Presidente de la Comisión Nacional de Especialidades de Enfermería de Salud Mental.

Contacto: jotacarretero@gmail.com

Fecha de recepción: 02 de mayo de 2018 / Fecha de aceptación: 30 de mayo de 2018

Nuestra historia como enfermeras y enfermeros de salud mental es tan larga como la propia historia del ser humano y, por ese motivo, resulta complejo realizar un análisis de nuestros orígenes. No obstante, existen pruebas históricas que narran ciertos cuidados brindados a personas con problemas de salud mental en todas las épocas de la humanidad, aunque probablemente sea durante el último siglo cuando se ha determinado en mayor medida la asistencia a la salud mental que conocemos hoy día.

Entre los primeros documentos en los que hay constancia de regulación de la atención a las personas con enfermedad mental en nuestro país, encontramos el Real Decreto de 12 de mayo de $1885^{1}$, que regulaba el régimen y gobierno interior del manicomio de Santa Isabel de Leganés, y el Real Decreto de 19 de mayo de $1885^{2}$, que establecía las reglas para el ingreso de los dementes en los manicomios del Estado, provinciales y particulares.

A comienzos del siglo xx, se produjo un aumento de la morbilidad psiquiátrica, promovida, entre otros factores, por la crisis económica de 1921 posterior a la primera guerra mundial. En la España de 1922, había acogidos en centros psiquiátricos 42 de cada 100.000 habitantes, mientras que en 1874 la cifra era de 18 de cada 100.000 habitantes, es decir, se produjo un aumento de más del $50 \%$ de personas institucionalizadas en apenas 50 años. Se crearon nuevos manicomios y se aumentó la capacidad de los ya existentes, todo ello sin que se mejoraran la atención ni los cuidados, ya que el concepto de enfermo mental llevaba implícita la etiqueta de incurable y, por lo tanto, destinado a un internamiento permanente en las instituciones psiquiátricas ${ }^{3}$.

Con la publicación del Decreto del 3 de julio de 1931, se suprimió la reclusión definitiva, y es a través de la publicación de la Orden Ministerial de 16 de mayo de $1932^{4}$ donde se definió claramente que el personal encargado del cuidado de las personas con enfermedad mental debía poseer el título de enfermero psiquiátrico. Para obtener este título, era necesario presentarse a los exámenes que se realizaban anualmente, además de realizar estudios prácticos y teóricos en un establecimiento psiquiátrico, público o privado, con una duración de dos años.

Todos los logros conseguidos en la etapa anterior fueron truncados por la contienda civil. Tras la Ley de Coordinación Hospitalaria de $1962^{5}$, se realizaron inversiones muy elevadas destinadas a la construcción de nuevos hospitales psiquiátricos. Conviene también resaltar que, según el censo de hospitales de 1978, alrededor del 1 \% de la población se encontraba ingresada de forma permanente en hospitales psiquiátricos, y de éstos sólo el 50\% presentaba una patología propiamente psiquiátrica ${ }^{6}$.

Por otro lado, la tendencia de unificación de las profesiones auxiliares sanitarias determinó su unificación con una única titulación, la de ayudante técnico sanitario (ATS). Se fue desarrollando un cuadro de especialidades para los ATS, creándose la especialidad de enfermería psiquiatría el 22 de noviembre de 1970 a través del Real Decreto $3193 / 1970^{7}$.

Posteriormente, y como consecuencia de una crisis general en relación a la atención psiquiátrica, en la que, por un lado, se evidenció la ineficacia de los manicomios para el tratamiento y rehabilitación de las personas con enferme- 
dad mental y, por el otro, se impusieron las tendencias que promovían la humanización de la asistencia sanitaria a los pacientes mentales ${ }^{8}$, se incluyó la asistencia y hospitalización psiquiátrica como prestación de la Seguridad Social en el año 1985, con un nuevo paradigma teórico que dio pie a la Reforma Psiquiátrica. Estas directrices fueron recogidas en la Ley General de Sanidad de $1986^{9}$, promoviendo que los servicios de salud mental dieran respuesta mediante un enfoque rehabilitador, de mantenimiento en la comunidad, atención individualizada y trabajo en equipo. La aplicación de esta ley tuvo distintos desarrollos y grados de éxito en las diferentes comunidades autónomas, debido a las transferencias de las competencias sobre organización y asistencia sanitarias.

De esta forma, los usuarios, en principio destinados a centros de día que pretendían descentralizar la asistencia, pero que apenas contaban con presupuesto y apoyo social, quedan al cuidado de unos familiares que no tenían ni los medios ni el conocimiento necesario ${ }^{8}$.

Recalcar a su vez una serie de fechas clave para la profesión, incluidas dentro de nuestro pasado más reciente. Es imprescindible mencionar la integración de enfermería en la Universidad a través del Real Decreto $2128 / 1977^{10}$ y de la creación, diez años más tarde, concretamente el 30 de junio de 1998, del título de Enfermera Especialista en Salud Mental, a través de la orden ministerial que desarrolla el Real Decreto 992/1987 ${ }^{11}$. A partir de ese año 1998, se han formado enfermeras especialistas por la vía EIR, inicialmente de un año de duración y, desde la entrada en vigor del Real Decreto, de 11 de mayo de $2011^{12}$, de dos años de duración. Con la publicación del Real Decreto 450/2005, de 22 de $\mathrm{abril}^{13}$, se suprimieron las especialidades anteriores, estableciendo las siete especialidades enfermeras vigentes en la actualidad, convalidando la antigua especialidad de enfermería psiquiátrica por la nueva de salud mental. La declaración de Bolonia, en 1999 ${ }^{14}$, marcó el inicio del proceso de convergencia hacia el Espacio Europeo de Educación Superior, que finalmente se hizo realidad en el año 2010, creando una oportunidad para la mejora de la calidad de la enseñanza superior , también en nuestra disciplina.

Según el informe sobre profesionales de cuidados de enfermería Oferta-Necesidad 2010-2025, publicado por el Ministerio de Sanidad, Servicios Sociales e Igualdad ${ }^{15}$, se convalidaron un total de 2030 títulos de enfermeras especialistas en psiquiatría por el de enfermeras especialistas en salud mental. Este real decreto también contemplaba la posibilidad de solicitar el acceso al título de especialista por vía excepcional, aunque para ello los aspirantes debían cumplir una serie de requisitos relacionados con la experiencia y formación que, en el caso de enfermería de salud mental, después de una prórroga inicial, debían de cumplirse antes del 22 de agosto de 2008. Por esta vía obtuvieron el título de enfermera especialista en salud mental un total de 2763 enfermeras ${ }^{15}$.

Desde entonces, la forma de acceder a una plaza de formación de especialista en enfermería de salud mental es a través de la realización de la prueba objetiva anual, única y simultánea, de carácter estatal para todas las especialidades enfermeras, conocida como EIR, que da acceso a una plaza de formación sanitaria especializada.

En los últimos 25 años se han convocado en todo el territorio estatal más de 13.000 plazas de formación sanitaria especializada, de las cuales un $21 \%$ han sido para enfermeras especialistas en salud mental. Desde el año 1998, en el que se instauró la vía EIR para salud mental, se han convocado 2678 plazas de formación sanitaria especializada ${ }^{15}$, lo que ha supuesto en términos económicos una inversión de más de 120 millones de euros.

Si tenemos en cuenta el número de enfermeras que convalidaron su título de especialistas en psiquiatría, así como aquellas que lo obtuvieron por la vía excepcional, y sumando a esa cifra las 20 promociones de enfermeras especialistas formadas por la vía EIR, existen casi 7500 enfermeras especialistas en salud mental a nivel estatal. A esta cifra habría que descontar, lógicamente, los profesionales que se han ido jubilando en los últimos años.

Valorando todo el esfuerzo que supone la formación de las enfermeras especialistas en salud mental, tanto a nivel personal como de las Unidades Docentes Multiprofesionales y los docentes y/o tutores que forman parte de ellas, es inevitable el pararse a pensar en el para qué de todo este esfuerzo.

Pues bien, a pesar de que el Ministerio de Sanidad recomendó tener creada en cada una de las comunidades autónomas, bien la categoría de enfermera especialista en salud mental, bien la categoría genérica de especialista ${ }^{16}$, en 
mayo de 2016, el desarrollo e implementación real de la especialidad de enfermería de salud mental en cada una de las comunidades autónomas resulta muy variopinto:

- Galicia, Castilla y León, Cantabria, País Vasco, Aragón, Extremadura, Murcia, la Comunidad Valenciana y las Islas Baleares tienen creada la categoría de enfermera especialista genérica o la específica de enfermera especialista en salud mental, así como un catálogo específico de puestos de trabajo. De hecho, algunas de ellas ya han llevado a cabo ofertas públicas de empleo específicas para enfermeras especialistas en salud mental.

- En Navarra, Madrid, Castilla-La Mancha, Andalucía y las Islas Canarias existe o bien la categoría de enfermera especialista genérica o la específica de salud mental. En Andalucía y Navarra existe una bolsa específica para enfermeras de salud mental, aunque no es un requisito imprescindible el ser especialista, solamente se precisa tener experiencia. Castilla-La Mancha y Madrid han anunciado que van a catalogar las plazas de enfermeras especialistas en salud mental a corto plazo.

- En el caso de Asturias, La Rioja y Cataluña, a día de hoy, todavía no han creado la categoría de enfermera especialista ni se han catalogado las plazas donde debe haber una enfermera especialista en salud mental. En el caso de Asturias, existe una bolsa para enfermeras que tengan experiencia en salud mental, aunque no es un requisito imprescindible ser especialista.

Socialmente hay una demanda de enfermeras especialistas en salud mental que acompañen a las personas, familias y grupos en su proceso, tanto puntualmente como durante periodos de larga duración, con un enfoque rehabilitador y de recuperación, rentabilizando los recursos invertidos. Por estos motivos, se hace imprescindible la creación de la categoría de enfermera de salud mental en todas y cada unas de las comunidades autónomas, así como la definición del catálogo específico de puestos de trabajo que deben desempeñar.

\section{Referencias bibliográficas}

1. Real Decreto 12 de mayo de 1885. Gaceta de Madrid. Disponible en: https://www.boe.es/datos/pdfs/BOE/1885/135/A0044700450.pdf. Consultado el: 13 de enero de 2018.

2. Real Decreto 19 de mayo de 1885. Gaceta de Madrid. Disponible en: https://www.boe.es/datos/pdfs/BOE/1885/141/A0051100511.pdf. Consultado el: 13 de enero de 2018.

3. Carretero J, Megías F, Cuesta D. Actualización en salud mental para enfermería. Formannova; 2015. ISBN: 978-84-606-6273-0.

4. Orden Ministerial de 16 de mayo de 1932. Disponible en: https://www.boe.es/datos/pdfs/BOE/1932/141/A0133401335.pdf. Consultado el: 13 de enero de 2018.

5. Ley $37 / 1962$, de 21 de julio, sobre hospitales.Disponible en: https://www.boe.es/boe/dias/1962/07/23/pdfs/A1026910271.pdf. Consultado el: 01 de febrero de 2018.

6. García J, Espino A, Lara L. La psiquiatría en la España de fin de siglo. Un estudio sobre la reforma psiquiátrica y las nuevas formas de atención en salud mental. Ed. Díaz de Santos. 1998. ISBN 847978-341-9.

7. Decreto $3192 / 1970$, de 22 de octubre, por el que crea la especialidad de «Neurologia» en los estudios de Ayudantes Técnicos Sanitarios. Disponible en:

https://www.boe.es/boe/dias/1970/11/09/pdfs/A1811718118.pdf. Consultado el: 01 de febrero de 2018.

8. Madoz-Gúrpide A, Ballesteros JC, Leira M, García E. Necesidad de un nuevo enfoque en la atención integral a los pacientes con trastorno mental grave treinta años después de la reforma psiquiátrica. Rev. Esp. Salud Publica vol.91 Madrid 2017 Epub 18-Ene-2017

9. Ley 14/1986, de 25 de abril, General de Sanidad.Disponible en: https://www.boe.es/buscar/act.php?id=BOE-A-1986-10499. Consultado el: 13 de enero de 2018.
10. Real Decreto 2128/1977, de 23 de julio, sobre integración en la Universidad de las Escuelas de Ayudantes Técnicos Sanitarios como Escuelas Universitarias de Enfermería.Disponible en: https://www.boe.es/buscar/doc.php?id=BOE-A-1977-20006. Consultado el: 01 de febrero de 2018.

11. Real Decreto 992/1987, de 3 de julio, por el que se regula la obtención del título de Enfermero especialista. Disponible en: https://www.boe.es/buscar/doc.php?id=BOE-A-1987-17866. Consultado el: 01 de febrero de 2018.

12. Orden SPI/1356/2011, de 11 de mayo, por la que se aprueba y publica el programa formativo de la especialidad de Enfermería de Salud Mental. Disponible en: https://www.boe.es/boe/dias/2011/05/24/pdfs/BOE-A-20119081.pdf. Consultado el: 01 de febrero de 2018.

13. Real Decreto $450 / 2005$, de 22 de abril, sobre especialidades de Enfermería. Disponible en:

https://www.boe.es/buscar/act.php?id=BOE-A-2005-7354. Consultado el: 03 de febrero de 2018.

14. Declaración de Bolonia. Disponible en: http://www.eees.es/pdf/Declaracion_Bolonia.pdf. Consultado el: 10 de enero de 2018.

15. López JA, Carbajo P, Giménez A, De Jorge M, Camarero C. Ministerio de Sanidad, Política Social e Igualdad. Dirección General de Ordenación Profesional, Cohesión del SNS y Alta Inspección. Informe sobre profesionales de cuidados de enfermería OfertaNecesidad 2010-2025 publicado por el Ministerio de Sanidad, Servicios Sociales e Igualdad (2012).

16. Propuesta de cronograma para la creación, implantación y dotación de plazas de las categorías de enfermeros-as especialistas en los distintos servicios de salud. Ministerio de Sanidad, Servicios Sociales e Igualdad (2014). 